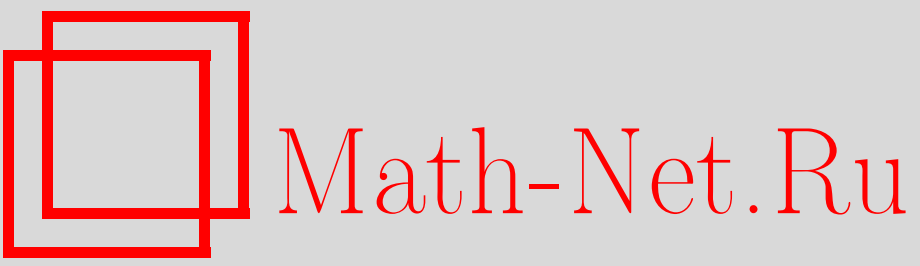

А. Дегасперис, С. В. Манаков, П. М. Сантини, Смешанные задачи для линейных и солитонных уравнений в частных производных, ТМФ, 2002, том 133, номер 2, 184-201

DOI: https://doi.org/10.4213/tmf389

Использование Общероссийского математического портала Math-Net.Ru подразумевает, что вы прочитали и согласны с пользовательским соглашением

http: //www . mathnet.ru/rus/agreement

Параметры загрузки:

IP : 54.197 .217 .227

26 апреля 2023 г., 14:13:27 
ТЕОРЕТИЧЕСКАЯ

И МАТЕМАТИЧЕСКАЯ

ФИЗИКА

Том 133, № 2

ноябрь, 2002

(C) 2002 г. $\quad$ А. Дегасперис ${ }^{*}$, С. В. Манаков ${ }^{\dagger}$, П. М. Сантини*

\section{СМЕШАННЫЕ ЗАДАЧИ ДЛЯ ЛИНЕЙНЫХ И СОЛИТОННЫХ УРАВНЕНИЙ В ЧАСТНЫХ ПРОИЗВОДНЫХ}

Рассматриваются эволюционные уравнения в частных производных для волн с дисперсией как в линейном, так и в нелинейном интегрируемом случаях и даются формулировки соответствующих смешанных задач в спектральном пространстве. Предложен метод решения, основанный на фиксации произвола в выборе граничных данных с помощью наложения подходящих ограничений на функциональное пространство и на комплексную область допустимых спектральных переменных. Подход проиллюстрирован на примере линейного уравнения Шредингера на ограниченных и полуограниченных $n$-мерных областях и на примере нелинейного уравнения Шредингера на полупрямой.

Ключевые слова: солитоны, интегрируемость, граничные условия.

\section{1. ВВЕДЕНИЕ}

Смешанные задачи (С3) для уравнений в частных производных (УрЧП) играют важную роль в физических приложениях и, можно даже сказать, в естественных науках вообще. Хорошо известно, что основная трудность при исследовании СЗ для линейных и нелинейных интегрируемых УрЧП состоит в наличии неизвестных граничных значений для соответствуюших уравнений, что характерно для любого метода решения задачи.

В данной работе сначала формулируется СЗ для линейных и нелинейных интегрируемых УрЧП в спектральном пространстве, а затем дается метод решения, называемый подходом "устранения по ограничению" (УПО), основанный на стратегии устранения неизвестных граничных условий с помощью наложения подходяших ограничений на функциональное пространство и на комплексную область определения весьма сложных спектральных функций. Этот подход возник из метода функции Грина $(\Phi Г)$, который по сути является аналогом этого метода в конфигурационном пространстве в случае линейной задачи, и из последних находок авторов для нелинейных задач.

\footnotetext{
*Dipartimento di Fisica, Università di Roma "La Sapienza"; Istituto Nazionale di Fisica Nucleare, Sezione di Roma, Roma, Italy.

E-mail: antonio.degasperis@roma1.infn.it;paolo.santini@roma1.infn.it

${ }^{\dagger}$ Институт теоретической физики им. Л. Д. Ландау, Москва, Россия. E-mail: manakov@itp.ac.ru
} 
Работа имеет следуюшую структуру. В разделе 2 рассматривается СЗ для линейного УрЧП с постоянными коэффициентами. Вводя подходяшее преобразование Фурье в этой задаче и устанавливая его свойства аналитичности, можно сначала выразить преобразование Фурье решения в терминах преобразований Фурье от известных и неизвестных начальных и граничных значений с помошью формулы Грина. Затем описывается подход УПО, в котором систематически используется стратегия устранения неизвестных граничных значений, что в итоге приводит к подходящему спектральному представлению решения, носитель которого может в конце концов даже стать дискретным, а не непрерывным, как в случае общего исходного интеграла Фурье. Этот метод оказывается довольно мошным, что показано на примере решения СЗ для уравнения Шредингера в прямоугольном ящике и квадранте.

В разделе 3 стратегия УПО применяется к солитонным уравнениям. После задания подходящего спектрального преобразования $S(k, t)$ для данной СЗ процедура УПО применяется для устранения неизвестных граничных значений из уравнений, определяюших $S(k, t)$, и для задания $S(k, t)$ с помошью нелинейного интегрального уравнения. Этот подход, представленный для модельного примера нелинейного уравнения Шредингера (НУШ) на полупрямой, может быть в принципе обобщен на случай отрезка. Так как в методе УПО используются спектральные переменные, сопряженные пространственным переменным, то этот метод работает одинаково хорошо как при пространственно-временных преобразованиях, так и для только пространственных преобразований. В линейном случае оказывается, что проще всего работать с пространственно-временным преобразованием Фурье, в то время как в нелинейном случае предпочтительным оказывается пространственное преобразование, а именно широко используемое (спектральное) преобразование обратной задачи рассеяния. Наконец, будет показана эквивалентность С3 для солитонных уравнений на полупрямой некоторым задачам со специальными начальными и граничными данными на всей прямой. Важным следствием этой эквивалентности является то, что из хорошо известного асимптотического поведения солитонных уравнений на всей прямой можно немедленно получить асимптотическое поведение СЗ на полупрямой с убывающими граничными условиями. Обзор литературы, посвященной исследованию данной проблемы, содержится в разделе 4.

Результаты данной статьи - это расширенный вариант материалов, представленных авторами на европейской конференции "NEEDS 2001" и на семинаре "Граничные задачи" - первом и последнем мероприятиях семестра "Интегрируемые системы", который имел место в период с июля по декабрь 2001 г. в Институте Исаака Ньютона в г. Кембридже.

\section{2. ПОДХОД УСТРАНЕНИЯ ПО ОГРАНИЧЕНИЮ. ЛИНЕЙНЫЙ СЛУЧАЙ}

Известно, что преобразование Фурье хорошо приспособлено для решения СЗ для линейных УрЧП в пространствах $\mathbb{R}^{n+1}$ с убываюшими граничными данными: 


$$
\begin{aligned}
\mathcal{L}\left(\nabla, \frac{\partial}{\partial t}\right) u(\mathbf{x}, t) & =f(\mathbf{x}, t), \quad \mathbf{x}=\left(x_{1}, \ldots, x_{n}\right) \in \mathbb{R}^{n}, \quad t>0, \\
u(\mathbf{x}, 0) & =u_{0}(\mathbf{x}), \quad u(\mathbf{x}, t) \rightarrow 0, \quad|\mathbf{x}| \rightarrow \infty,
\end{aligned}
$$

где $\boldsymbol{\nabla}=\left(\partial / \partial x_{1}, \ldots, \partial / \partial x_{n}\right), \mathcal{L}$ - оператор в частных производных с постоянными коэффициентами, $u(\mathbf{x}, t)$ - неизвестное поле, $f(\mathbf{x}, t)$ - заданная сила и $u_{0}(\mathbf{x})$ - заданное начальное условие.

В данном разделе будет описан весьма эффективный подход (в пространстве Фурье) для решения более сложных С3, заданных на ограниченных или полуограниченных пространственных областях $V$,

$$
\mathcal{L}\left(\nabla, \frac{\partial}{\partial t}\right) u(\mathbf{x}, t)=f(\mathbf{x}, t), \quad \mathbf{x} \in V \subset \mathbb{R}^{n}, \quad t>0,
$$

с граничными условиями типа Дирихле или Неймана, или со смешанными граничными условиями на $\partial V$.

2.1. Преобразование Фурье и его свойства. Естественное преобразование Фурье, связанное с пространственно-временной областью $\mathcal{D}=V \otimes(0, \infty)$ (обозначаемое в дальнейшем $\left.\mathrm{FT}_{\mathcal{D}}\right)$, по определению имеет вид

$$
\widehat{F}(\mathbf{k}, q)=\int_{\mathcal{D}} d \mathbf{x} d t e^{-i(\mathbf{k} \cdot \mathbf{x}+q t)} F(\mathbf{x}, t)
$$

для любой гладкой функции $F(\mathbf{x}, t),(\mathbf{x}, t) \in \mathcal{D}$, в предположении, что $F(\mathbf{x}, t) \rightarrow 0, t \rightarrow$ $\infty$ достаточно быстро; здесь $\mathbf{k}=\left(k_{1}, \ldots, k_{n}\right) \in \mathbb{R}^{n}, q \in \mathbb{R}$ и $\mathbf{k} \cdot \mathbf{x}=\sum_{j} k_{j} x_{j}$. Соответствуюшее обратное преобразование

$$
F(\mathbf{x}, t) \chi_{\mathcal{D}}(\mathbf{x}, t)=\int_{\mathbb{R}^{n+1}} \frac{d \mathbf{k} d q}{(2 \pi)^{n+1}} e^{i(\mathbf{k} \cdot \mathbf{x}+q t)} \widehat{F}(\mathbf{k}, q)
$$

воспроизводит $F(\mathbf{x}, t)$ в области $\mathcal{D}$ и обрашается в ноль вне этой области. Здесь $\chi \mathcal{D}(\mathbf{x}, t)$ представляет собой характеристическую функцию области $\mathcal{D}: \chi_{\mathcal{D}}(\mathbf{x}, t)=1, \quad(\mathbf{x}, t) \in$ $\mathcal{D}, \chi_{\mathcal{D}}(\mathbf{x}, t)=0,(\mathbf{x}, t) \notin \mathcal{D}\left(\right.$ тем самым $\chi_{\mathcal{D}}(\mathbf{x}, t)=\chi_{V}(\mathbf{x}) H(t)$, где $H(t)-$ стандартная функция Хэвисайда (функция ступеньки)).

Если указанная область пространства оказывается полным пространством, $V=\mathbb{R}^{n}$, то $\mathrm{FT}_{\mathcal{D}}(3)$ определено в области $\mathcal{A}=\mathbb{R}^{n} \otimes \overline{\mathcal{I}}_{q}$, где $\overline{\mathcal{I}}_{q}$ - замыкание нижней полуплоскости $\mathcal{I}_{q}$ переменной $q$, аналитично при $q \in \mathcal{I}_{q} \forall \mathbf{k} \in \mathbb{R}^{n}$ и имеет правильное асимптотическое поведение при больших $|q|$ в области аналитичности. Если пространственная область $V$ компактна, то $\mathrm{FT}_{\mathcal{D}}$ имеет сильные свойства аналитичности по всем переменным Фурье: это преобразование определено в области $\mathcal{A}=\mathcal{C}^{n} \otimes \overline{\mathcal{I}}_{q}$, аналитично при $q \in \mathcal{I}_{q}, \forall \mathbf{k} \in \mathcal{C}^{n}$, представляет собой целую функцию при каждом комплексном $k_{j}$, $j=1, \ldots, n, \forall q \in \overline{\mathcal{I}}_{q}$ и обладает правильным асимптотическим поведением при больших $(\mathbf{k}, q)$ в областях аналитичности. Если область пространства полуограниченна, то аналитичность по фурье-переменным $k_{j}, j=1, \ldots, n$, имеет место в открытых областях комплексной плоскости, зависящих от геометрических характеристик области $V$. 
Для того чтобы выразить $\mathrm{FT}_{\mathcal{D}}$ решения в терминах $\mathrm{FT}_{\mathcal{D}}$ для вынужденных и начальных и граничных условий используются хорошо известная формула Грина (тождество Грина)

$$
b \mathcal{L} a-a \tilde{\mathcal{L}} b=\operatorname{div} J(\mathbf{x}, t)
$$

и ее интегральное выражение - знаменитое интегральное тождество Грина:

$$
\int_{\mathcal{D}}(b \mathcal{L} a-a \tilde{\mathcal{L}} b) d \mathbf{x} d t=\int_{\partial \mathcal{D}} J(\mathbf{x}, t) \cdot \boldsymbol{\nu} d \sigma
$$

получаемое с помошью интегрирования формулы $(5)$ по области $\mathcal{D}$ с последуюшим использованием формулы дивергенции. В уравнении (5) $\tilde{\mathcal{L}}$ - это оператор, формально сопряженный оператору $\mathcal{L}: \tilde{\mathcal{L}}=\mathcal{L}(-\nabla,-\partial / \partial t), J(\mathbf{x}, t)-(n+1)$-мерное векторное поле, $\operatorname{div}-(n+1)$-мерный оператор дивергенции, а $a(\mathbf{x}, t)$ и $b(\mathbf{x}, t)$ - произвольные функции. В уравнении (6) элемент $d \sigma-$ это элемент гранишы гиперповерхности и $\boldsymbol{\nu}$ - внешний единичный нормальный вектор этой гиперповерхности. Заметим, что если заданы оператор $\mathcal{L}$, его формально сопряженный $\tilde{\mathcal{L}}$ и две произвольные функции $a$ и $b$, то всегда существует $(n+1)$-мерное векторное поле $J(\mathbf{x}, t)$, удовлетворяющее формуле Грина $(5)$, и оно может быть алгоритмически найдено. Это поле оказывается линейньм по $a$, $b$ и их частным производным вплоть до порядка $N-1$, если только оператор $\mathcal{L}$ имеет порядок $N$.

Ввиду произвольности функций $a$ и $b$ из формул (5) и (6) можно извлечь довольно много полезной информации о граничной задаче; при частном выборе

$$
a=u(\mathbf{x}, t), \quad b=\frac{e^{-i(\mathbf{k} \cdot \mathbf{x}+q t)}}{\mathcal{L}(i \mathbf{k}, i q)}
$$

где $\mathcal{L}(i \mathbf{k}, i q)$ - собственное значение оператора $\mathcal{L}$, отвечающее собственной функции $e^{i(\mathbf{k} \cdot \mathbf{x}+q t)}$, векторное поле $J$ принимает следуюший вид:

$$
J=e^{-i(\mathbf{k} \cdot \mathbf{x}+q t)} J^{\prime}(\mathbf{x}, t ; \mathbf{k}, q) / \mathcal{L}(i \mathbf{k}, i q)
$$

При этом из интегрального тождества Грина (6) следует, что $\mathrm{FT}_{\mathcal{D}}$ решения может быть выражено в терминах $\mathrm{FT}_{\mathcal{D}}$ (или, возможно, в терминах обобщенных преобразований $Ф у-$ рье) от силовых факторов и от всех начальных и граничных данных:

$$
\hat{u}(\mathbf{k}, q)=\frac{\hat{f}(\mathbf{k}, q)-\int_{\partial \mathcal{D}} e^{-i(\mathbf{k} \cdot \mathbf{x}+q t)} J^{\prime}(\mathbf{x}, t ; \mathbf{k}, q) \cdot \boldsymbol{\nu} d \sigma}{\mathcal{L}(i \mathbf{k}, i q)}=: \frac{\widehat{\mathcal{N}}(\mathbf{k}, q)}{\mathcal{L}(i \mathbf{k}, i q)}, \quad(\mathbf{k}, q) \in \mathcal{A} .
$$

В обшем случае выражение $\mathcal{L}(i \mathbf{k}, i q)$, стояшее в знаменателе этого уравнения, представляет собой целую и даже в большинстве случаев полиномиальную функцию от всех своих комплексных аргументов; нули этой функции могут лежать на вешественной прямой. Таким образом, до того как вычислять обратное преобразование Фурье, необходимо регуляризовать эту функцию:

$$
\mathcal{L}(i \mathbf{k}, i q) \rightarrow \mathcal{L}_{\text {reg }}(i \mathbf{k}, i q)
$$


т.е. надо сдвинуть сингулярности с вещественной оси и добиться того, чтобы они лежали вне области $\mathcal{A}$.

Обратное преобразование Фурье (4) задает соответствуюшее представление Фурье для решения:

$$
U(\mathbf{x}, t)=u(\mathbf{x}, t) \chi_{\mathcal{D}}(\mathbf{x}, t)=\int_{\mathbb{R}^{n+1}} \frac{d \mathbf{k} d q}{(2 \pi)^{n+1}} e^{i(\mathbf{k} \cdot \mathbf{x}+q t)} \frac{\widehat{\mathcal{N}}(\mathbf{k}, q)}{\mathcal{L}_{\mathrm{reg}}(i \mathbf{k}, i q)}, \quad(\mathbf{x}, t) \in \mathbb{R}^{n+1}
$$

Ясно, что этого еще недостаточно, так как в случае обшего положения правая часть уравнения (8) все еше зависит как от известных, так и от неизвестных граничных данHыIx.

2.2. Устранение по ограничению в пространстве Фурье. Традиционные способы решения СЗ для линейных УрЧП состоят в нахождении подходяших стратегий для устранения неизвестных граничных условий из представлений для решений. Эти методы основаны на знаменитом подходе ФГ, в котором:

1) сначала строится интегральное представление Грина

$$
u(\mathbf{x}, t)=\int_{\mathcal{D}} d \mathbf{x}^{\prime} d t^{\prime} \tilde{g}\left(\mathbf{x}, t ; \mathbf{x}^{\prime}, t^{\prime}\right) f\left(\mathbf{x}^{\prime}, t^{\prime}\right)-\int_{\partial \mathcal{D}} J\left(\mathbf{x}, t ; \mathbf{x}^{\prime}, t^{\prime}\right) \cdot \boldsymbol{\nu}_{x^{\prime}} d \sigma_{x^{\prime}}, \quad(\mathbf{x}, t) \in \mathcal{D}
$$

для решения СЗ (2). Это представление является другим приложением формулы (6), отвечаюшим выбору: $a(\mathbf{x}, t)=u(\mathbf{x}, t)$ и $b(\mathbf{x}, t)=\tilde{g}\left(\mathbf{x}^{\prime}, t^{\prime} ; \mathbf{x}, t\right)$, где $\tilde{g}-$ любая функция Грина оператора $\tilde{\mathcal{L}}_{x}: \tilde{\mathcal{L}}_{x} \tilde{g}\left(\mathbf{x}^{\prime}, t^{\prime} ; \mathbf{x}, t\right)=\delta\left(\mathbf{x}-\mathbf{x}^{\prime}\right) \delta\left(t-t^{\prime}\right),(\mathbf{x}, t),\left(\mathbf{x}^{\prime}, t^{\prime}\right) \in \mathcal{D} ;$

2 ) затем используется произвол в выборе функции $\tilde{g}$ и строится именно та функция Грина, которая позволяет устранить вклады, зависящие от неизвестных граничных значений. Метод разложения по собственным функциям также основан на идее устранения, и этот метод оказывается эквивалентным подходу $Ф$, в котором строится набор собственных функций оператора $\mathcal{L}$ с такими граничными условиями, которые позволяют опять-таки устранить неизвестные граничные значения. Оба эти подхода имеют функционально-аналитическую природу.

В данном разделе будет показано, как стратегия устранения может быть естественным образом введена, если использовать представление пространства Фурье, определенного в (3) (стратегия устранения в спектральном пространстве уже была успешно применена при исследовании солитонных уравнений на полупрямой (см. раздел 4)).

Уравнение (8), заданное на подходяшей области $\mathcal{A} \subset \mathcal{C}^{n+1}$, обычно проявляет несколько свойств симметрии, являюшихся следствием структуры $\mathcal{L}$ и геометрии пространственной области $V$. Первая и наиболее важная часть метода состоит в том, чтобы систематически использовать эти свойства симметрий в пространстве Фурье для построения такого линейного оператора $\mathcal{E}$, который в правой части уравнения (8) уничтожает вклады, приходящие от неизвестных граничных значений, содержашихся в числителе $\widehat{\mathcal{N}}(\mathbf{k}, q)$, и при этом оставляет знаменатель $\mathcal{L}(i \mathbf{k}, i q)$ нетронутым:

$$
\mathcal{E} \hat{u}(\mathbf{k}, q)=\mathcal{E}\left(\frac{\widehat{\mathcal{N}}}{\mathcal{L}}\right)(\mathbf{k}, q)=\{\text { только известные величины }\}, \quad(\mathbf{k}, q) \in \mathcal{A}^{\prime} \subset \mathcal{A} .
$$


Процедура устранения, задаваемая линейным оператором $\mathcal{E}$, позволяет построить $\mathcal{E} \hat{u}$ в подпространстве $\mathcal{A}^{\prime}$ исходного пространства, на котором был определен оператор $\hat{u}$. Таким образом, необходимо выяснить, достаточна ли полученная информация для того, чтобы восстановить решение $u$, т.е. задает ли $\mathcal{E} \hat{u}$ обратимое спектральное преобразование в области $\mathcal{D}$.

Используя уравнение (3), можно определить это новое спектральное преобразование следующим образом:

$$
\begin{aligned}
\mathcal{E} \hat{u}(\mathbf{k}, q)= & \int_{\mathcal{D}} d \mathbf{x} d t \tilde{\varphi}_{\mathbf{k}, q}(\mathbf{x}, t) u(\mathbf{x}, t), \quad(\mathbf{k}, q) \in \mathcal{A}^{\prime}, \\
& \tilde{\varphi}_{\mathbf{k}, q}(\mathbf{x}, t):=\mathcal{E}\left(e^{-i(\mathbf{k} \cdot \mathbf{x}+q t)}\right) .
\end{aligned}
$$

Это преобразование оказывается обратимым, если существует собственная функция $\varphi_{\mathbf{k}, q}(\mathbf{x}, t)$ оператора $\mathcal{L}$, обладаюшая свойством полноты:

$$
\sum_{(\mathbf{k}, q) \in \mathcal{A}^{\prime}} \varphi_{\mathbf{k}, q}(\mathbf{x}, t) \tilde{\varphi}_{\mathbf{k}, q}\left(\mathbf{x}^{\prime}, t^{\prime}\right)=\delta\left(t-t^{\prime}\right) \delta\left(\mathbf{x}-\mathbf{x}^{\prime}\right), \quad(\mathbf{x}, t),\left(\mathbf{x}^{\prime}, t^{\prime}\right) \in \mathcal{D} .
$$

В этом случае обратное преобразование позволяет построить функцию $\widetilde{U}(\mathbf{x}, t)$, определенную во всем пространстве-времени, которая совпадает с решением в области $\mathcal{D}$ :

$$
\begin{aligned}
& \widetilde{U}(\mathbf{x}, t)=\sum_{\mathbf{k}, q} \varphi_{\mathbf{k}, q}(\mathbf{x}, t) \mathcal{E}\left(\frac{\widehat{\mathcal{N}}}{\mathcal{L}}\right)(\mathbf{k}, q), \quad(\mathbf{x}, t) \in \mathbb{R}^{n} \otimes(0, \infty), \\
& \widetilde{U}(\mathbf{x}, t)=u(\mathbf{x}, t), \quad(\mathbf{x}, t) \in \mathcal{D} .
\end{aligned}
$$

В уравнениях (14) и (15) символ $\sum_{\mathbf{k}, q}$ обозначает суммирование и/или интегрирование в зависимости от природы области $\mathcal{A}^{\prime}$ определения нового спектрального преобразования. Подводя итог, приходим к следующим выводам.

Хотя прямое преобразование, с которого начиналось рассмотрение, представляет собой преобразование Фурье $\hat{u}$, задаваемое формулой (3), процедура УПО, примененная к этому преобразованию, приводит к новому прямому преобразованию Фурье $\mathcal{E} \hat{u}(13)$, обратное к которому, как правило, существенно отличается от преобразования (4). Отсюда следует, что воспроизведенная функция $\widetilde{U}(\mathbf{x}, t)$ совпадает с решением $u(\mathbf{x}, t)$ в области $\mathcal{D}$, но не обращается в нуль вне области $\mathcal{D}$ и наследует свойства симметрии собственной функции $\varphi_{\mathbf{k}, q}(\mathbf{x}, t)$.

2.3. Поясняющий пример. В этом разделе подход УПО применяется к СЗ (2), отвечающей оператору

$$
\begin{gathered}
\mathcal{L}=i \frac{\partial}{\partial t}+\Delta, \quad \Delta=\nabla \cdot \nabla=\sum_{j=1}^{n} \frac{\partial^{2}}{\partial x_{j}^{2}} \\
V=\left\{\mathbf{x}: 0 \leqslant x_{j} \leqslant L_{j}, j=1, \ldots, n\right\}
\end{gathered}
$$

т.е. С3 решается для $(n+1)$-мерного уравнения Шредингера в области пространства, представляюшей собой $n$-мерный прямоугольный ящик. 
Тогда получим

$$
\begin{gathered}
\tilde{\mathcal{L}}=-i \frac{\partial}{\partial t}+\Delta, \quad J=(i a b, b \nabla a-a \nabla b), \\
\mathcal{L}(i \mathbf{k}, i q)=-\left(q+k^{2}\right) \Rightarrow \mathcal{L}_{\text {reg }}(i \mathbf{k}, i q)=-\left(q+k^{2}-i 0\right),
\end{gathered}
$$

где $k^{2}=\mathbf{k} \cdot \mathbf{k}$. Уравнения (8) и (17) задают следующее выражение для преобразования Фурье решения в терминах преобразований Фурье силовых факторов и всех начальных и граничных данных:

$$
\begin{gathered}
\hat{u}(\mathbf{k}, q)=-\frac{\widehat{\mathcal{N}}(\mathbf{k}, q)}{q+k^{2}-i 0}, \quad(\mathbf{k}, q) \in \mathcal{A}, \\
\widehat{\mathcal{N}}(\mathbf{k}, q):=\hat{f}(\mathbf{k}, q)+i \hat{u}_{0}(\mathbf{k})+\sum_{j=1}^{n}\left\{\left[\widehat{w}_{0 j}\left(\mathbf{k}_{j}, q\right)+i k_{j} \hat{v}_{0 j}\left(\mathbf{k}_{j}, q\right)\right]-\right. \\
-e^{\left.-i k_{j} L_{j}\left[\widehat{w}_{L j}\left(\mathbf{k}_{j}, q\right)+i k_{j} \hat{v}_{L j}\left(\mathbf{k}_{j}, q\right)\right]\right\}}
\end{gathered}
$$

(в своей области определения $\left.\mathcal{A}=\mathcal{C}^{n} \otimes \overline{\mathcal{I}}_{q}\right)$, где $\hat{f}, \hat{u}_{0}, \hat{v}_{0 j}, \hat{v}_{L j}, \widehat{w}_{0 j}$ и $\widehat{w}_{L j}$ суть преобразования Фурье силовых и начальных и граничных данных:

$$
\begin{gathered}
u_{0}(\mathbf{x})=\left.u(\mathbf{x}, t)\right|_{t=0}, \quad v_{0 j}\left(\mathbf{x}_{j}, t\right)=\left.u(\mathbf{x}, t)\right|_{x_{j}=0}, \quad v_{L j}\left(\mathbf{x}_{j}, t\right)=\left.u(\mathbf{x}, t)\right|_{x_{j}=L_{j}}, \\
w_{0 j}\left(\mathbf{x}_{j}, t\right)=\left.\frac{\partial u}{\partial x_{j}}(\mathbf{x}, t)\right|_{x_{j}=0}, \quad w_{L j}\left(\mathbf{x}_{j}, t\right)=\left.\frac{\partial u}{\partial x_{j}}(\mathbf{x}, t)\right|_{x_{j}=L_{j}} ;
\end{gathered}
$$

т.е., например,

$$
\hat{u}_{0}(\mathbf{k})=\int_{V} d \mathbf{x} e^{-i \mathbf{k} \cdot \mathbf{x}} u_{0}(\mathbf{x}), \quad \hat{v}_{0 j}\left(\mathbf{k}_{j}, q\right)=\int_{0}^{\infty} d t \int_{V_{j}} d \mathbf{x}_{j} e^{-i\left(\mathbf{k}_{j} \cdot \mathbf{x}_{j}+q t\right)} v_{0 j}\left(\mathbf{x}_{j}, t\right) .
$$

В уравнениях (18)-(20) и ниже полагается, что $\mathbf{x}_{j}=\left(x_{1}, \ldots, \check{x}_{j}, \ldots, x_{n}\right) \in \mathbb{R}^{n-1}, \mathbf{k}_{j}=$ $\left(k_{1}, \ldots, \check{k}_{j}, \ldots, k_{n}\right) \in \mathbb{R}^{n-1}$,

$$
\int_{V_{j}} d \mathbf{x}_{j}=\int_{0}^{L_{1}} d x_{1} \ldots\left(\int_{0}^{L_{j}} d x_{j}\right)^{\vee} \cdots \int_{0}^{L_{n}} d x_{n}
$$

где символ ${ }^{\vee}$ означает, что отмеченная им величина должна быть опущена.

Конечно, в случае, когда все вышеприведенные граничные данные известны, решение $u$ задается формулой (10):

$$
\begin{aligned}
U(\mathbf{x}, t)= & u(\mathbf{x}, t) \chi_{V}(\mathbf{x}) H(t)=-\int_{\mathbb{R}^{n+1}} \frac{d \mathbf{k} d q}{(2 \pi)^{n+1}} e^{i(\mathbf{k} \cdot \mathbf{x}+q t)} \frac{\widehat{\mathcal{N}}(\mathbf{k}, q)}{q+k^{2}-i 0}= \\
= & -\int_{\mathbb{R}^{n+1}} \frac{d q d \mathbf{k}}{(2 \pi)^{n+1}} e^{i(\mathbf{k} \cdot \mathbf{x}+q t)} \frac{\hat{f}(\mathbf{k}, q)}{q+k^{2}-i 0}+H(t) \int_{\mathbb{R}^{n}} \frac{d \mathbf{k}}{(2 \pi)^{n}} e^{i\left(\mathbf{k} \cdot \mathbf{x}-k^{2} t\right)} \hat{u}_{0}(\mathbf{k})+ \\
& +\sum_{j=1}^{n} \int_{\mathbb{R}^{n-1}} \frac{d \mathbf{k}_{j}}{(2 \pi)^{n-1}} e^{i \mathbf{k}_{j} \cdot \mathbf{x}_{j}} \times \\
& \times \int_{\gamma} \frac{d k_{j}}{2 \pi i}\left\{e^{i\left(k_{j}\left|x_{j}\right|-k^{2} t\right)}\left[\widehat{w}_{0 j}\left(\mathbf{k}_{j},-k^{2}\right)+i \operatorname{sign}\left(x_{j}\right) k_{j} \hat{v}_{0 j}\left(\mathbf{k}_{j},-k^{2}\right)\right]-\right. \\
& \left.-e^{i\left(k_{j}\left|x_{j}-L_{j}\right|-k^{2} t\right)}\left[\widehat{w}_{L j}\left(\mathbf{k}_{j},-k^{2}\right)+i \operatorname{sign}\left(x_{j}-L_{j}\right) k_{j} \hat{v}_{L j}\left(\mathbf{k}_{j},-k^{2}\right)\right]\right\},
\end{aligned}
$$


где $d \mathbf{k}_{j}=d k_{1} \ldots \check{d k_{j}} \ldots d k_{n}$ и $\gamma=(i \infty, 0) \cup(0, \infty)$.

Ввиду специальных свойств четности преобразований Фурье в формулах (18), в дальнейшем будут использоваться так называемые операторы четности:

$$
\Delta_{ \pm}=\prod_{l=1}^{n}\left(1 \pm \hat{\sigma}_{l}\right), \quad \Delta_{ \pm}^{(j)}=\prod_{\substack{l=1 \\ l \neq j}}^{n}\left(1 \pm \hat{\sigma}_{l}\right),
$$

где $\hat{\sigma}_{j}-$ операторы инволюций $\hat{\sigma}_{j}: k_{j} \rightarrow-k_{j}$.

Допустим, что решается задача Дирихле. Тогда применение оператора четности $\Delta_{-}$ к выражениям (18) позволяет убрать все величины $\widehat{w}_{0}$ :

$$
\begin{aligned}
\Delta_{-} \hat{u}(\mathbf{k}, q)= & -\left(\Delta_{-}\left[\hat{f}(\mathbf{k}, q)+i \hat{u}_{0}(\mathbf{k})\right]+2 i \sum_{j=1}^{n} k_{j} \Delta_{-}^{(j)} \hat{v}_{0 j}\left(\mathbf{k}_{j}, q\right)+\right. \\
& \left.+2 i \sum_{j=1}^{n}\left[\sin \left(k_{j} L_{j}\right) \Delta_{-}^{(j)} \widehat{w}_{L j}\left(\mathbf{k}_{j}, q\right)-k_{j} \cos \left(k_{j} L_{j}\right) \Delta_{-}^{(j)} \hat{v}_{L j}\left(\mathbf{k}_{j}, q\right)\right]\right) \times \\
& \times\left(q+k^{2}-i 0\right)^{-1}, \quad(\mathbf{k}, q) \in \mathcal{A} .
\end{aligned}
$$

Теперь, для того чтобы убрать также и все величины $\widehat{w}_{L}$, надо ограничить возможные значения $k_{j}$ на дискретный набор $k_{j}=h_{j}:=\pi m_{j} / L_{j}, m_{j} \in \mathbb{Z}$, так что исходная область $\mathcal{A}$ оказывается в конце концов ограниченной на следуюшую область:

$$
(\mathbf{k}, q) \in \mathcal{A}^{\prime}=\left\{(\mathbf{h}, q) ; q \in \overline{\mathcal{I}}_{q}, \mathbf{h}=\left(h_{1}, \ldots, h_{n}\right), h_{j}=\frac{\pi m_{j}}{L_{j}}, m_{j} \in \mathbb{Z}, j=1, \ldots, n\right\} .
$$

Таким образом, УПО с помощью оператора $\mathcal{E}$ в этом примере дает

$$
\mathcal{E} \cdot=\int_{\mathbb{R}^{n}} d \mathbf{k} \delta(\mathbf{k}-\mathbf{h}) \Delta_{-} \cdot
$$

и его применение к функции $\hat{u}$ приводит к нужному результату:

$$
\begin{aligned}
\mathcal{E} \hat{u}(\mathbf{k}, q)= & \left(\Delta_{-} \hat{u}\right)(\mathbf{h}, q)=-\left\{\Delta_{-}\left[\hat{f}(\mathbf{h}, q)+i \hat{u}_{0}(\mathbf{h})\right]+\right. \\
& \left.+2 i \sum_{j=1}^{n} h_{j} \Delta_{-}^{(j)}\left[\hat{v}_{0 j}\left(\mathbf{h}_{j}, q\right)-(-1)^{m_{j}} \hat{v}_{L j}\left(\mathbf{h}_{j}, q\right)\right]\right\}\left(q+h^{2}-i 0\right)^{-1},
\end{aligned}
$$

где $\mathbf{h}_{j}=\left(h_{1}, \ldots, \check{h}_{j}, \ldots, h_{n}\right)$ и $h^{2}=\mathbf{h} \cdot \mathbf{h}$. Преобразование $\mathcal{E} \hat{u}(\mathbf{k}, q)$, задаваемое процедурой УПО, представляет собой хорошо известное многомерное дискретное синусоидальное преобразование:

$$
\begin{gathered}
\Delta_{-} \hat{u}(\mathbf{h}, q)=\int_{\mathcal{D}} d t d \mathbf{x} \tilde{\varphi}_{\mathbf{h}, q}(\mathbf{x}, t) u(\mathbf{x}, t) \\
\tilde{\varphi}_{\mathbf{h}, q}(\mathbf{x}, t):=\Delta_{-}\left(e^{-i(\mathbf{h} \cdot \mathbf{x}+q t)}\right)=(-2 i)^{n} e^{-i q t} \prod_{l=1}^{n} \sin \left(h_{l} x_{l}\right) .
\end{gathered}
$$


Чтобы построить обратное к этому преобразованию, используются формулы

$$
\varphi_{\mathbf{h}, q}(\mathbf{x}, t)=\frac{i^{n}}{L_{1} \ldots L_{n}} \frac{e^{i q t}}{2 \pi} \prod_{l=1}^{n} \sin \left(h_{l} x_{l}\right), \quad \sum_{\mathbf{k}, q}=\int_{\mathbb{R}} d q \sum_{m_{1}=1}^{\infty} \cdots \sum_{m_{n}=1}^{\infty}
$$

так что разложение $(15)$ задает функцию $\widetilde{U}(\mathbf{x}, t)$, определенную во всем пространстве-времени и совпадающую с решением $u(\mathbf{x}, t) \mathrm{C} 3$ при ограничении $(\mathbf{x}, t) \in \mathcal{D}$ :

$$
\begin{aligned}
\widetilde{U}(\mathbf{x}, t)= & \frac{i^{n}}{L_{1} \ldots L_{n}} \sum_{m_{1}=1}^{\infty} \cdots \sum_{m_{n}=1}^{\infty} \prod_{l=1}^{n} \sin \left(h_{l} x_{l}\right) \int_{\mathbb{R}} \frac{d q}{2 \pi} e^{i q t} \times \\
& \times \Delta_{-}\left(\frac{\widehat{\mathcal{N}}(\mathbf{h}, q)}{\mathcal{L}(i \mathbf{h}, i q)}\right), \quad(\mathbf{x}, t) \in \mathbb{R}^{n+1}, \\
\widetilde{U}(\mathbf{x}, t)= & u(\mathbf{x}, t), \quad(\mathbf{x}, t) \in \mathcal{D} .
\end{aligned}
$$

Из уравнения (29) следует, что благодаря свойствам симметрии функции $\varphi_{\mathbf{h}, q}(\mathbf{x}, t)$ функция $\widetilde{U}$, в свою очередь, будет нечетным $(2 L)$-периодическим расширением решения вне области $\mathcal{D}$ и будет задавать дискретное тригонометрическое (с помощью синусов) фурье-преобразование решения:

$$
\begin{aligned}
u(\mathbf{x}, t)= & -\frac{i^{n}}{L_{1} \ldots L_{n}}\left\{\sum_{\mathbf{h}} \prod_{l=1}^{n} \sin \left(h_{l} x_{l}\right)\left[\int_{\mathbb{R}} \frac{d q}{2 \pi} e^{i q t} \frac{\Delta_{-} \hat{f}(\mathbf{h}, q)}{q+h^{2}-i 0}-e^{-i h^{2} t} \Delta_{-} \hat{u}_{0}(\mathbf{h})\right]+\right. \\
& +\sum_{j=1}^{n} L_{j} \sum_{\mathbf{h}_{j}} \prod_{l \neq j} \sin \left(h_{l} x_{l}\right) \int_{\gamma} \frac{d k_{j}}{\pi} \frac{i k_{j} e^{-i\left(k_{j}^{2}+\mathbf{h}_{j} \cdot \mathbf{h}_{j}\right) t}}{\sin \left(k_{j} L_{j}\right)} \Delta_{-}^{(j)} \times \\
& \times\left[\sin k_{j}\left(L_{j}-x_{j}\right) \hat{v}_{0 j}\left(\mathbf{h}_{j},-k_{j}^{2}-\mathbf{h}_{j} \cdot \mathbf{h}_{j}\right)+\right. \\
& \left.\left.+\sin \left(k_{j} x_{j}\right) \hat{v}_{L j}\left(\mathbf{h}_{j},-k_{j}^{2}-\mathbf{h}_{j} \cdot \mathbf{h}_{j}\right)\right]\right\}, \quad(\mathbf{x}, t) \in \mathcal{D}
\end{aligned}
$$

где

$$
\sum_{\mathbf{h}} \equiv \sum_{m_{1}=1}^{\infty} \ldots \sum_{m_{n}=1}^{\infty}, \quad \sum_{\mathbf{h}_{j}} \equiv \sum_{m_{1}=1}^{\infty} \cdots\left(\sum_{m_{j}=1}^{\infty}\right)^{\vee} \cdots \sum_{m_{n}=1}^{\infty}
$$

и интеграл $\int_{\gamma} d k_{j}$ регуляризован условием, что сингулярности $\pi m_{j} / L_{j}, m_{j} \in \mathbb{Z}^{+}$, сдвинуты во внешность первого квадранта.

Задача Неймана может быть решена аналогичным образом с использованием дискретного преобразования косинусов:

$$
\begin{gathered}
\mathcal{E} \cdot=\int_{\mathbb{R}^{n}} d \mathbf{k} \delta(\mathbf{k}-\mathbf{h}) \Delta_{+} \cdot \\
\tilde{\varphi}_{\mathbf{h}, q}(\mathbf{x}, t):=\Delta_{+}\left(e^{-i(\mathbf{h} \cdot \mathbf{x}+q t)}\right)=2^{n} e^{-i q t} \prod_{l=1}^{n} \cos \left(h_{l} x_{l}\right), \\
\varphi_{\mathbf{h}, q}(\mathbf{x}, t)=\frac{1}{2^{n} L_{1} \ldots L_{n}} \frac{e^{i q t}}{2 \pi} \prod_{l=1}^{n} \cos \left(h_{l} x_{l}\right), \quad \sum_{\mathbf{k}, q}=\int_{\mathbb{R}} d q \sum_{m_{1}=-\infty}^{\infty} \ldots \sum_{m_{n}=-\infty}^{\infty},
\end{gathered}
$$


и с помощью дискретного представления решения через косинусы:

$$
\begin{aligned}
u(\mathbf{x}, t)= & -\frac{1}{2^{n} L_{1} \ldots L_{n}}\left\{\sum_{\mathbf{h}} \prod_{l=1}^{n} \cos \left(h_{l} x_{l}\right)\left[\int_{\mathbb{R}} \frac{d q}{2 \pi} e^{i q t} \frac{\Delta_{+} \hat{f}(\mathbf{h}, q)}{q+h^{2}-i 0}-e^{-i h^{2} t} \Delta_{+} \hat{u}_{0}(\mathbf{h})\right]-\right. \\
& -2 \sum_{j=1}^{n} L_{j} \sum_{\mathbf{h}_{j}} \prod_{l \neq j} \cos \left(h_{l} x_{l}\right) \int_{\gamma} \frac{d k_{j}}{\pi} \frac{e^{-i\left(k_{j}^{2}+\mathbf{h}_{j} \cdot \mathbf{h}_{j}\right) t}}{\sin \left(k_{j} L_{j}\right)} \Delta_{+}^{(j)} \times \\
& \times\left[\cos k_{j}\left(L_{j}-x_{j}\right) \widehat{w}_{0 j}\left(\mathbf{h}_{j},-k_{j}^{2}-\mathbf{h}_{j} \cdot \mathbf{h}_{j}\right)-\right. \\
& \left.\left.-\cos \left(k_{j} x_{j}\right) \widehat{w}_{L j}\left(\mathbf{h}_{j},-k_{j}^{2}-\mathbf{h}_{j} \cdot \mathbf{h}_{j}\right)\right]\right\}, \quad(\mathbf{x}, t) \in \mathcal{D},
\end{aligned}
$$

где

$$
\sum_{\mathbf{h}} \equiv \sum_{m_{1}=-\infty}^{\infty} \ldots \sum_{m_{n}=-\infty}^{\infty}, \quad \sum_{\mathbf{h}_{j}} \equiv \sum_{m_{1}=-\infty}^{\infty} \ldots\left(\sum_{m_{j}=-\infty}^{\infty}\right)^{\vee} \ldots \sum_{m_{n}=-\infty}^{\infty},
$$

а интеграл $\int_{\gamma} d k_{j}$ регуляризован так же, как и в формуле $(30)$.

Используя теорему о свертке, можно сразу получить из уравнений (30) и (33) для решения интегральное представление Грина (11), которое отвечает соответственно следующим запаздывающим функциям Грина задач Дирихле и Неймана:

$$
\begin{aligned}
G_{\mathrm{RD}}\left(\mathbf{x}, t ; \mathbf{x}^{\prime}, t^{\prime}\right)= & \frac{2^{n} i}{L_{1} \ldots L_{n}} H\left(t-t^{\prime}\right) \times \\
& \times \sum_{m_{1}=1}^{\infty} \cdots \sum_{m_{n}=1}^{\infty} e^{-i h^{2}\left(t-t^{\prime}\right)} \prod_{l=1}^{n} \sin \left(h_{l} x_{l}\right) \sin \left(h_{l} x_{l}^{\prime}\right), \\
G_{\mathrm{RN}}\left(\mathbf{x}, t ; \mathbf{x}^{\prime}, t^{\prime}\right)= & \frac{-i}{L_{1} \ldots L_{n}} H\left(t-t^{\prime}\right) \times \\
& \times \sum_{m_{1} \in \mathbb{Z}} \cdots \sum_{m_{n} \in \mathbb{Z}} e^{-i h^{2}\left(t-t^{\prime}\right)} \prod_{l=1}^{n} \cos \left(h_{l} x_{l}\right) \cos \left(h_{l} x_{l}^{\prime}\right) .
\end{aligned}
$$

В случае полуограниченных областей подходяшее спектральное преобразование, получаемое в подходе УПО, имеет, вообше говоря, непрерывный носитель. Например, для задачи Дирихле уравнения Шредингера в $n$-квадранте

$$
V=\left\{\mathbf{x} \in \mathbb{R}^{n}: x_{j} \geqslant 0, j=1, \ldots, n\right\}
$$

получим, что оператор устранения $\mathcal{E}=\Delta_{-}$, который сужает область определения до $\mathcal{A}^{\prime}=\mathbb{R}^{n} \otimes \overline{\mathcal{I}}_{q}$, приводит к непрерывному тригонометрическому (с помощью синусов) преобразованию Фурье:

$$
\begin{gathered}
\tilde{\varphi}_{\mathbf{k}, q}(\mathbf{x}, t)=(-2 i)^{n} e^{-i q t} \prod_{l=1}^{n} \sin \left(k_{l} x_{l}\right), \\
\varphi_{\mathbf{k}, q}(\mathbf{x}, t)=\frac{e^{i(\mathbf{k} \cdot \mathbf{x}+q t)}}{(2 \pi)^{n+1}}, \quad \sum_{\mathbf{k}, q}=\int_{\mathbb{R}} d q \int_{\mathbb{R}^{n}} d \mathbf{k}
\end{gathered}
$$


и к соответствующему непрерывному многомерному синус-фурье-преобразованию решения:

$$
\begin{aligned}
u(\mathbf{x}, t)=- & \int_{\mathbb{R}^{n+1}} \frac{d q d \mathbf{k}}{(2 \pi)^{n+1}} e^{i(\mathbf{k} \cdot \mathbf{x}+q t)} \frac{\Delta_{-} \hat{f}(\mathbf{k}, q)}{q+k^{2}-i 0}+\int_{\mathbb{R}^{n}} \frac{d \mathbf{k}}{(2 \pi)^{n}} e^{i\left(\mathbf{k} \cdot \mathbf{x}-k^{2} t\right)} \Delta_{-} \hat{u}_{0}(\mathbf{k})+ \\
& +\sum_{j=1}^{n} \int_{\mathbb{R}^{n-1}} \frac{d \mathbf{k}_{j}}{(2 \pi)^{n-1}} \int_{\gamma} \frac{d k_{j}}{\pi} e^{i\left(\mathbf{k} \cdot \mathbf{x}-k^{2} t\right)} k_{j} \Delta_{-}^{(j)} \hat{v}_{0 j}\left(\mathbf{k}_{j},-k^{2}\right), \quad(\mathbf{x}, t) \in \mathcal{D} .
\end{aligned}
$$

Из приведенных модельных примеров следует, что метод УПО оказывается весьма эффективным в пространстве Фурье и, возможно, более простым по сравнению с подходом функции Грина, который представляет собой его аналог в конфигурационном пространстве. Сравнение этих двух методов устранения в примерах, в которых подход ФГ неприменим, будет проведено в отдельной публикации.

\section{3. ПОДХОД УСТРАНЕНИЯ ПО ОГРАНИЧЕНИЮ. НЕЛИНЕЙНЫЙ СЛУЧАЙ}

В этом разделе рассмотрим СЗ, связанную с нелинейными эволюционными УрЧП, которые интегрируются с помошью (спектрального) метода обратной задачи рассеяния. Ограничимся рассмотрением наглядного примера применения данного метода решения к НУШ

$$
i q_{t}+q_{x x}+c|q|^{2} q=0, \quad q=q(x, t),
$$

где $c$ - произвольный вещественный параметр. Однако этот метод также хорошо применим и к другим $(1+1)$-мерным солитонным уравнениям (например, к уравнению Кортевега-де Фриза (Кд $\Phi))$. Более того, рассмотрение далее будет ограничено на те решения уравнения (39), которые лежат в первом квадранте плоскости $(x, t)$, а именно на решения на полупрямой $0 \leqslant x \leqslant \infty$ при $t \geqslant 0$. Решение однозначно задается совокупностью начальных данных $q(x, 0)=q_{0}(x)$ и граничных данных

$$
f(t)=a_{1} v(t)+a_{2} w(t), \quad t \geqslant 0,
$$

где введены обозначения

$$
v(t)=q(0, t), \quad w(t)=q_{x}(0, t)
$$

Здесь $a_{1}$ и $a_{2}$ - заданные вешественные константы, и при $a_{2}=0$ или $a_{1}=0$ мы получаем соответственно граничную задачу Дирихле или Неймана. Таким образом, задача состоит в построении решения $q(x, t)$ уравнения $(39)$, когда начальные значения $q_{0}(x)$ и граничные условия $f(t)$ являются заданными функциями в соответствующем функциональном пространстве (можно предположить, что они представляют собой комплекснозначные функции, быстро убываюшие при $x \rightarrow \infty$ и $t \rightarrow \infty)$.

Главное свойство НУШ (39) - это то, что это уравнение есть условие интегрируемости для следующей пары $(2 \times 2)$-матричных линейных обыкновенных дифференциальных уравнений (ОДУ):

$$
\Psi_{x}=\left(i k \sigma_{3}+Q\right) \Psi, \quad \Psi_{t}=2 i k^{2}\left[\sigma_{3}, \Psi\right]+M \Psi
$$


где $\sigma_{3}=\operatorname{diag}(1,-1)$ и

$$
Q(x, t)=\left(\begin{array}{cc}
0 & -c \bar{q}(x, t) \\
q(x, t) & 0
\end{array}\right), \quad M(x, t, k)=2 k Q-i \sigma_{3} Q_{x}+i Q^{2} \sigma_{3}
$$

Решение $\Psi(x, t, k)$ этих уравнений задается асимптотическим условием

$$
\Psi(x, t, k) e^{-i k x \sigma_{3}} \rightarrow I, \quad x \rightarrow \infty
$$

которое однозначно определяет матрицу рассеяния $S(k, t)$ стандартным образом, а именно как граничное значение

$$
S(k, t)=\Psi(0, t, k)
$$

Нам понадобятся следуюшие хорошо известные утверждения, которые оказываются весьма полезными с точки зрения излагаемого ниже метода. Матричное решение $\Psi$, а потому (см. (45)) и матрица рассеяния $S$ имеют единичный детерминант

$$
\operatorname{det} \Psi(x, t, k)=\operatorname{det} S(k, t)=1 \text {. }
$$

Более того, свойство

$$
Q^{\dagger}=-C Q C^{-1}, \quad C:=\left(\begin{array}{cc}
1 & 0 \\
0 & c
\end{array}\right)
$$

матрицы $Q$ (см. (43)) приводит к соответствующим свойствам

$$
\Psi^{\dagger}(x, t, k)=C \Psi^{-1}(x, t, \bar{k}) C^{-1}, \quad S^{\dagger}(k, t)=C S^{-1}(\bar{k}, t) C^{-1}
$$

для решений Йоста $\Psi$ и матрицы рассеяния $S$ (индекс ${ }^{\dagger}$ обозначает эрмитово сопряжение). Следствием этого оказывается то, что матрицу рассеяния $S$ удобно параметризовать с помошью двух функций $\alpha(k, t)$ и $\beta(k, t)$ в соответствии с определением

$$
S(k)=\left(\begin{array}{cc}
\alpha(k) & -c \bar{\beta}(\bar{k}) \\
\beta(k) & \bar{\alpha}(\bar{k})
\end{array}\right) .
$$

Что касается $k$-зависимости (здесь комплексная спектральная переменная $k$ играет ту же роль, что и переменная Фурье в случае линейной задачи), то в этом случае функции $\alpha(k, t)$ и $\beta(k, t)$ оказываются аналитическими функциями в верхней полуплоскости ( $\operatorname{Im} k>0)$, имеющими следующее асимптотическое поведение:

$$
\alpha(k, t)=1+O\left(k^{-1}\right), \quad \beta(k, t)=O\left(k^{-1}\right)
$$

при больших $|k|$. Наконец, напомним, что из таких аналитических свойств функций $\alpha(k, t)$ и $\beta(k, t)$ вытекает способ решения обратной задачи, т.е. $S(k, t) \rightarrow Q(x, t)$ при любом фиксированном $t \geqslant 0 ;$ при этом основные уравнения обратной задачи, которые здесь не приводятся, имеют либо вид интегральных уравнений типа Коши по переменной $k$, либо, эквивалентно, они записываются как интегральное уравнение типа Марченко по переменной $x$. 
Обратимся теперь к эволюции по времени. Здесь вершиной спектрального метода является эволюционное уравнение для матрицы рассеяния (см. (42) и (45))

$$
S_{t}=2 i k^{2}\left[\sigma_{3}, S\right]+Z(k, t) S,
$$

которое следует из того, что зависимости матрицы $Z(k, t)$ от двух граничных функций $v(t)$ и $w(t)$ разделяются (см. (41)) в соответствии со следующими выражениями:

$$
\begin{aligned}
Z(k, t) & =2 k V(t)-i \sigma_{3} W(t)+i V^{2}(t) \sigma_{3} \\
V(t) & =Q(0, t), \quad W(t)=Q_{x}(0, t) .
\end{aligned}
$$

Отсюда вытекает, что эволюционное уравнение (51) не может быть непосредственно проинтегрировано, что дало бы матрицу рассеяния, необходимую для воспроизведения $Q(x, t)$ через решения обратной задачи. Поскольку в нашей постановке заданы только граничные данные (40), в полной аналогии с методом устранения в линейном случае необходимо ввести новую матрицу

$$
\widetilde{S}(k, t)=A^{-1}(k) S^{-1}(-k, t) A(k) S(k, t), \quad A(k):=a_{1} I+2 i k a_{2} \sigma_{3},
$$

которая обладает двумя важными свойствами. Во-первых, ее детерминант единичен (см. (46)) и ее асимптотическое значение при $|k| \rightarrow \infty$ оказывается единичной матрицей:

$$
\operatorname{det} \widetilde{S}(k, t)=1, \quad \widetilde{S}(k, t)=I+O\left(k^{-1}\right)
$$

Во-вторых, эта матрица удовлетворяет эволюционному уравнению, которое содержит только лишь данное граничное условие (40), а именно,

$$
\begin{aligned}
\widetilde{S}_{t} & =2 i k^{2}\left[\sigma_{3}, \widetilde{S}\right]+4 k A^{-1}(k) S^{-1}(-k, t) F(t) S(k, t), \\
F(t) & =a_{1} V(t)+a_{2} W(t)=\left(\begin{array}{cc}
0 & -c \bar{f}(t) \\
f(t) & 0
\end{array}\right) .
\end{aligned}
$$

Хотя этот шаг весьма важен, его недостаточно для построения решения данной задачи, так как в эволюционном уравнении (первое уравнение в (55)) все еще присутствует неизвестная матрица рассеяния $S(k, t)$. Поэтому надо найти способ связать между собой матрицы $S(k, t)$ и $\widetilde{S}(k, t)$. Соотношение $S(k, t) \rightarrow \widetilde{S}(k, t)$ тривиально, так как оно задается собственно определением (53). Из этого соотношения следует начальное условие $\widetilde{S}(k, 0)$ при интегрировании эволюционного уравнения $(55)$, т.е. $Q(x, 0) \rightarrow \Psi(x, 0, k) \rightarrow$ $S(k, 0)=\Psi(0,0, k) \rightarrow \widetilde{S}(k, 0)$. Что касается обратного соотношения $\widetilde{S}(k, t) \rightarrow S(k, t)$, то вместо него можно задать задачу Римана-Гильберта, которая в конце концов приводит к интегральному уравнению типа Коши. Переписав уравнение $(53)$ в виде $A(k) \times$ $S(k, t)=S(-k, t) A(k) \widetilde{S}(k, t)$ и заметив, что первый столбец матришы $S(k, t)(49)$ аналитичен в верхней полуплоскости и имеет асимптотическое поведение (50), можно найти два связанных интегральных уравнения для величин $\alpha(k, t)$ и $\beta(k, t)$ в терминах матрицы $\widetilde{S}(k, t)$, если использовать стандартную технику задачи Римана-Гильберта. Если 
предположить для простоты, что в верхней полуплоскости не содержится полюсов, и подставить вместо $\widetilde{S}(k, t)$ выражение для этой величины, полученное при формальном интегрировании эволюционного уравнения (55), окончательно получим два связанных нелинейных интегральных уравнения

$$
\begin{aligned}
& \alpha(k, t)=1+\frac{c}{2 \pi i} \int_{-\infty}^{\infty} \frac{d k^{\prime}}{k^{\prime}-(k+i 0)} e^{-4 i k^{\prime 2} t} h\left(k^{\prime}, t\right) \bar{\beta}\left(k^{\prime}, t\right), \\
& \beta(k, t)=-\frac{1}{2 \pi i} \int_{-\infty}^{\infty} \frac{d k^{\prime}}{k^{\prime}-(k+i 0)} e^{-4 i k^{\prime 2} t} h\left(k^{\prime}, t\right) \bar{\alpha}\left(k^{\prime}, t\right),
\end{aligned}
$$

нелинейность которых появляется за счет того, что функция $h(k, t)$ сама зависит от неизвестных функций $\alpha(k, t)$ и $\beta(k, t)$ через интегральное (относительно $t$ ) соотношение:

$$
\begin{aligned}
h(k, t)= & \left\{a(k) \alpha_{0}(k) \beta_{0}(-k)-a(-k) \alpha_{0}(-k) \beta_{0}(k)-\right. \\
& \left.-4 k \int_{0}^{t} d t^{\prime} e^{4 i k^{2} t^{\prime}}\left[f\left(t^{\prime}\right) \alpha\left(k, t^{\prime}\right) \alpha\left(-k, t^{\prime}\right)+c \bar{f}\left(t^{\prime}\right) \beta\left(k, t^{\prime}\right) \beta\left(-k, t^{\prime}\right)\right]\right\} \times \\
& \times\left\{a(-k) \alpha_{0}(-k) \bar{\alpha}_{0}(k)+c a(k) \beta_{0}(-k) \bar{\beta}_{0}(k)-\right. \\
& \left.-4 k c \int_{0}^{t} d t^{\prime}\left[f\left(t^{\prime}\right) \alpha\left(-k, t^{\prime}\right) \bar{\beta}\left(k, t^{\prime}\right)-\bar{f}\left(t^{\prime}\right) \bar{\alpha}\left(k, t^{\prime}\right) \beta\left(-k, t^{\prime}\right)\right]\right\}^{-1},
\end{aligned}
$$

где $a(k)=a_{1}+2 i k a_{2}$ и $\alpha_{0}$ и $\beta_{0}$ соответственно представляют собой известные начальные значения $\alpha(k, 0)$ и $\beta(k, 0)$.

Обсуждение этих весьма сложных уравнений и их применений в различных областях лежит за пределами данной статьи и будет опубликовано отдельно. Здесь же только заметим, что эта формулировка естественным образом выделяет так называемые линеаризуемые С3, а именно те, для которых граничное значение $f(t)$ исчезает (см. (40)), $f(t)=0$. Действительно, в этом случае функция ядра $h(k, t)(57)$ не зависит от неизвестных $\alpha(k, t)$ и $\beta(k, t)$ и уравнения (56) становятся линейными. Заметим, что, полагая $c=0$, можно устранить нелинейность во всех вышеприведенных формулах, так что уравнение (39) становится линейным уравнением Шредингера, и тогда из уравнений $(56),(57)$ следует, что $\alpha(k, t)=1$, в то время как $\beta(k, t)$ совпадает со стандартным явным выражением для преобразования Фурье решения $q(x, t)$.

Наконец, мы считаем полезным представить здесь подход к решению начальной задачи для НУШ (39), который отличается от описанного выше и все-таки оказывается эквивалентным ему, поскольку в итоге приводит к тем же уравнениям (56) и (57). Основное свойство этого подхода состоит в том, что в нем СЗ формулируется на всей прямой, т.е. для $x \in(-\infty, \infty)$, и что матрища $\widetilde{S}(k, t)$, определенная выражениями (53), допускает теперь спектральное описание в рамках стандартных прямой и обратной задач, связанных с уравнением Лакса - первым ОДУ в системе (42). Расплачиваться за введение этой, более знакомой формулировки приходится тем, что нелинейное УрЧП, 
которое теперь надо решить, - это НУШ с неоднородным источником вместо стандартного НУШ (39). Ниже вкратце описан этот подход с двумя ограничениями, обусловленными исключительно соображениями краткости: рассматриваются только граничные задачи Дирихле и Неймана; предполагается, что спектральные данные в любой момент времени $t \geqslant 0$ не содержат компоненты дискретного спектра.

Исходное наблюдение состоит в том, что если $q(x, t)$ - решение НУШ $(39)$ при $x \in$ $(0, \infty)$ и $t \geqslant 0$, то функция

$$
\tilde{q}(x, t)=q(x, t) H(x)-\eta q(-x, t) H(-x), \quad \eta= \pm 1,
$$

определенная при каждом вешественном значении переменной $x$, удовлетворяет УрЧП

$$
i \tilde{q}_{t}+\tilde{q}_{x x}+2 c|\tilde{q}|^{2} \tilde{q}=(1+\eta) v(t) \delta^{\prime}(x)+(1-\eta) w(t) \delta(x),
$$

где $\delta(x)$ - обобщенная функция типа дельта-функции Дирака, $\delta^{\prime}(x)$ - ее производная, a $v$ и $w$ определены условиями (41). Очевидно, что значения $\eta=1$ и $\eta=-1$ отвечают соответствуюшему выбору граничных условий Дирихле или Неймана для СЗ.

Как следует из спектрального метода, основанного на уравнениях Лакса, удобно переписать формулы (58) и (59) в матричном виде, если ввести недиагональную матрицу $2 \times 2($ cм. $(43))$

$$
\widetilde{Q}(x, t)=Q(x, t) H(x)-\eta Q(-x, t) H(-x), \quad \eta= \pm 1,
$$

и УрЧП

$$
i \widetilde{Q}_{t}-\sigma_{3}\left(\widetilde{Q}_{x x}-2 \widetilde{Q}^{3}\right)=\Sigma(x, t),
$$

которое, разумеется, эквивалентно уравнению (59), если только член с источником имеет вид (см. второе уравнение в системе (52))

$$
\Sigma(x, t)=-\sigma_{3}\left[(1+\eta) V(t) \delta^{\prime}(x)+(1-\eta) W(t) \delta(x)\right] .
$$

Спектральный подход к уравнению (61) основан на спектральном уравнении

$$
\tilde{\Psi}_{x}=\left(i k \sigma_{3}+\widetilde{Q}(x, t)\right) \tilde{\Psi}, \quad \tilde{\Psi}=\tilde{\Psi}(x, t, k),
$$

что вполне стандартно. Решение Йоста $\tilde{\Psi}$ задается асимптотическим условием (44), $\tilde{\Psi} e^{-i k x \sigma_{3}} \rightarrow I, x \rightarrow \infty$, с помошью которого можно явно выразить это решение через решение $\Psi(x, t, k)$, определенное выше на полупрямой,

$$
\tilde{\Psi}(x, t, k)=\Psi(x, t, k) H(x)+E \Psi(-x, t,-k) E \widetilde{S}(k, t) H(-x),
$$

где $E=\operatorname{diag}(1, \eta)$ и

$$
\widetilde{S}(k, t)=E S^{-1}(-k, t) E S(k, t)
$$

- это в точности та матрица рассеяния, которая определяется стандартным образом, а именно,

$$
\tilde{\Psi}(x, t, k) \rightarrow e^{i k x \sigma_{3}} \widetilde{S}(k, t), \quad x \rightarrow-\infty .
$$


Отметим, что эта матрища рассеяния $\widetilde{S}(k, t)$ (65) совпадает с матрищей (53) при $a_{2}=0$ в случае задачи Дирихле $(\eta=1)$ и при $a_{1}=0$ в случае задачи Неймана $(\eta=-1)$.

Следуя стандартной процедуре, теперь надо ввести другое решение Йоста уравнения (63),

$$
\tilde{\Phi}(x, t, k)=\tilde{\Psi}(x, t, k) \widetilde{S}^{-1}(k, t),
$$

и учесть тождество

$$
\widetilde{S}_{t}+2 i k^{2}\left[\widetilde{S}, \sigma_{3}\right]=i \int_{-\infty}^{\infty} d x \tilde{\Phi}^{-1}(x, t, k)\left[i \widetilde{Q}_{t}-\sigma_{3}\left(\widetilde{Q}_{x x}-2 \widetilde{Q}^{3}\right)\right] \tilde{\Psi}(x, t, k),
$$

которое вкупе с неоднородным УрЧП (61) влечет эволюционное уравнение для матришы рассеяния

$$
\widetilde{S}_{t}=2 i k^{2}\left[\sigma_{3}, \widetilde{S}\right]+i \int_{-\infty}^{\infty} d x \tilde{\Phi}^{-1}(x, t, k) \Sigma(x, t) \tilde{\Psi}(x, t, k) .
$$

Теперь легко показать, что, внося под знак интеграла в правой части этого уравнения выражения (67), (64) и (62), мы получим в точности эволюционное уравнение (55) для СЗ Дирихле или Неймана

$$
\widetilde{S}_{t}=2 i k^{2}\left[\sigma_{3}, \widetilde{S}\right]+2 k(1+\eta) S^{-1}(-k, t) V(t) S(k, t)-i(1-\eta) \sigma_{3} S^{-1}(-k, t) W(t) S(k, t) .
$$

В заключение заметим, что достижение предъявленного подхода, которьй мы называем "метод источника", состоит в том, что в этом подходе можно использовать преимушества более традиционного (спектрального) метода обратной задачи рассеяния на всей прямой. В частности, становится возможным исследовать поведение решения $q(x, t)$ для СЗ при больших временах, поскольку асимптотические выражения при условии, что граничные данные быстро убывают при $t \rightarrow \infty$, оказываются сравнительно легко вычислимыми, если рассматривается обычная спектральная теория на всей прямой.

\section{4. ЛИТЕРАТУРНЫЙ ОБЗОР}

Классическая идея устранения неизвестных граничных данных из представления для решения лежит в основе метода функции Грина [1], в котором сушественно используется метод отражений [2] при построении подходяшей функции Грина, т.е. той, которая позволяет устранить неизвестные граничные данные. Подход УПО, введенный в данной работе, представляет собой естественное и эффективное использование стратегии устранения, примененной в пространстве Фурье. Насколько нам известно, этот метод никогда не применялся ранее к исследованию линейных УрЧП.

Альтернативный метод, который авторы называют аналитическим подходом, также дает полезные результаты; основан он на сушествовании глобального соотношения, открытого Фокасом, и на его использовании при решении С3 [3]-[7]. Наш вклад в этот метод заключается в систематическом использовании аналитических свойств фурьепреобразований всех величин, встречаюшихся в формуле (8), с целью вывести набор 
аналитических ограничений, которых оказывается достаточно для того, чтобы выразить неизвестные граничные условия через известные, и для того, чтобы исследовать в обшем случае однозначную разрешимость СЗ [8].

За последние десятилетия были развиты разнообразные подходы к изучению задач с неизвестными граничными данными при исследовании СЗ для солитонных уравнений. В работе [9] Фокас ввел нелинейньй аналог преобразования синусов. В работе [10] Сабатье построил “локтевое рассеяние” в плоскости переменных $(x, t)$, для того чтобы исследовать уравнение КдФ на полупрямой, что привело к формулировке Гельфанда-Левитана-Марченко. В работах [3], [4] Фокас применил другой подход, основанный на одновременном спектральном преобразовании для переменных $x$ и $t$. Этот подход был строго обоснован в работах [11], [12] в применении к решению СЗ для солитонных уравнений на полупрямой. Этот подход позволяет найти строгое асимптотическое поведение решения [13] и естественным образом охватывает уже известные модели [14] линеаризуемых граничных задач. В работе [15] были предложены два альтернативных подхода к исследованию СЗ для солитонных уравнений на отрезке и на полупрямой. В первом методе неизвестные граничные данные были выражены через элементы матрицы рассеяния $S(k, t)$, что порождает нелинейное интегродифференциальное эволюционное уравнение на матрицу $S$. Во втором методе, который может рассматриваться как нелинейный аналог подхода УПО, развитого в разделе 2 , для $S$ было построено нелинейное эволюционное уравнение (55), которое уже не содержит неизвестных граничных данных и включает в себя естественным образом случай линеаризуемой СЗ. Некоторые интегрируемые случаи граничных условий для солитонных уравнений и их связь с симметриями были также исследованы Хабибуллиным и др. (см. [16] и ссылки в этой работе).

В некоторых необщих случаях солитонных уравнений, соответствующих сингулярным дисперсионным соотношениям, к которым относятся уравнения вынужденного рамановского рассеяния (ВРP) и уравнение синус-Гордон $(\mathrm{C} \Gamma)$ в координатах светового конуса, эволюционное уравнение для матрицы рассеяния не содержит неизвестных граничных условий. Уравнение СГ на полупрямой было исследовано с помощью $x$ - $t$-спектрального преобразования [3]; уравнения ВРР и СГ на полупрямой также исследовались с использованием более традиционного метода $x$-преобразований Леоном и Михайловым в работе [17] и Леоном и Спиро в работе [18]; $x$-спектральные данные, использованные в последнем подходе, удовлетворяют нелинейному эволюционному уравнению типа уравнения Риккати.

Помимо одновременного преобразования пространственно-временных переменных, все вышеприведенные подходы были основаны на традиционном методе обратной задачи рассеяния [19]. Спектральный формализм, позволяющий исследовать “нагруженные” солитонные уравнения (солитонные уравнения с заданной ненулевой правой частью), был развит несколькими авторами, особенно в связи с теорией возмущений (см., например, [20]).

Отметим, наконец, что СЗ и задача для $C$-интегрируемого нагруженного уравнения также рассматривались Абловицем, Калоджеро и Де Лилло [21]. 
Результаты, относящиеся к нелинейному случаю, включают в себя: 1) построение с помошью техники Римана-Гильберта интегрального уравнения (56), (57) для элементов матришы рассеяния в замкнутом виде, 2) постановку СЗ Дирихле и Неймана на полупрямой для солитонных уравнений, рассматриваемых как нагруженные начальные задачи на всей прямой. Эквивалентность задач на полупрямой и на всей прямой уже использовалась в работе [9], хотя подходящие уравнения для спектральных данных, найденные в работе [9], отличаются от уравнений, представленных в настоящей работе.

Благодарности. Настоящая работа была выполнена в течение нескольких визитов и встреч. Мы благодарим за финансовую поддержку РФФИ (грант № 01-01-00929), INTAS (грант № 99-1782), а также Университет Рима “Ла Сапенца” (Италия), Национальный институт ядерных исследований (римская секция), Институт теоретической физики им. Л. Д. Ландау (Москва, Россия) и Институт математических наук Исаака Ньютона (Кембридж, Великобритания).

\section{Список литературы}

[1] Ф. Морс, Г. Фешбах. Методы теоретической физики. М.: Изд-во иностр. лит., 1958.

[2] R. Terras, R. Swanson. J. Math. Phys. 1980. V. 21. P. 2140-2153.

[3] A. S. Fokas. Proc. Roy. Soc. Lond. A. 1997. V. 53. P. 1411.

[4] A. S. Fokas. J. Math. Phys. 2000. V. 41. P. 4188.

[5] A. S. Fokas. Proc. Roy. Soc. Lond. A. 2001. V. 457. P. 371

[6] A. S. Fokas, B. Pelloni. Math. Proc. Camb. Phil. Soc. 2001. V. 131. P. 521.

[7] Б. Пеллони. ТМФ. 2002. Т. 133. № 2. С. 327.

[8] A. Degasperis, S. V. Manakov, P. M. Santini. Initial-boundary value problems for linear PDEs: the analyticity approach. nlin.SI/0210058.

[9] A. S. Fokas. Inverse scattering transform on the half-line - the nonlinear analogue of the sine transform. In: Inverse Problems: An Interdisciplinary Study. Ed. P. C. Sabatier. London: Academic Press, 1987. P. 409.

[10] P. C. Sabatier. J. Math. Phys. 2000. V. 41. P. 414.

[11] A. S. Fokas, A. R. Its, L. Y. Sung. The nonlinear Schrödinger equation on the half-line. Preprint. Cambridge: Cambridge Univ. Press, 2001.

[12] A. S. Fokas. Integrable nonlinear evolution equations on the half-line. Commun. Math. Phys. (in print).

[13] A. S. Fokas, A. R. Its. Phys. Rev. Lett. 1992. V. 68. P. 3117.

[14] M. J. Ablowitz, H. Segur. J. Math. Phys. 1975. V. 16. P. 1054.

[15] А. Дегасперис, С. В. Манаков, П. М. Сантини. Письма в ЖЭТФ. 2001. Т. 74. С. 541.

[16] И. Т. Хабибуллин. ТМФ. 2002. Т. 130. № 1. С. 31.

[17] J. Leon, A. V. Mikhailov. Phys. Lett. A. 1999. V. 253. P. 33.

[18] J. Leon, A. Spire. J. Phys. A. 2001. V. 34. P. 7359.

[19] М. Дж. Абловии, Х. Сигур. Солитоны и метод обратной задачи. М.: Мир, 1987; В. E. Захаров, С. В. Манаков, С. П. Новиков, Л. П. Питаевский. Теория солитонов: Метод обратной задачи. М.: Наука, 1980.

[20] А. Ньюәлл. Обратное преобразование рассеяния. В сб.: Солитоны. Ред. Р. Буллаф, Ф. Кодри. М.: Мир, 1983. С. 193.

[21] F. Calogero, S. De Lillo. Inverse Problems. 1988. V. 4. P. L33-37; J. Math. Phys. 1991. V. 32. P. 99; M. J. Ablowitz, S. De Lillo. Phys. Lett. A. 1991. V. 156. P. 483. 